\title{
BMJ Open Stepped care approach for medial tibiofemoral osteoarthritis (STrEAMline): protocol for a randomised controlled trial
}

\author{
Sarah Rubia Robbins, ${ }^{1,2}$ Luciano Ricardo S Melo, ${ }^{1,2}$ Hema Urban, ${ }^{1,2}$ \\ Leticia A Deveza, ${ }^{1,2}$ Rebecca Asher, ${ }^{3}$ Victoria L Johnson, ${ }^{1,2}$ David J Hunter ${ }^{1,2}$
}

To cite: Robbins SR, Melo LRS, Urban $\mathrm{H}$, et al. Stepped care approach for medial tibiofemoral osteoarthritis (STrEAMline): protocol for a randomised controlled trial. BMJ Open 2017;7:e018495. doi:10.1136/ bmjopen-2017-018495

- Prepublication history for this paper is available online. To view these files, please visit the journal online (http://dx.doi org/10.1136/bmjopen-2017018495).

SRR and LRSM contributed equally.

Received 4 July 2017 Revised 9 November 2017 Accepted 13 November 2017

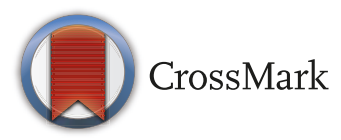

${ }^{1}$ Kolling Institute of Medical Research, Institute of Bone and Joint Research, University of Sydney, Sydney, Australia ${ }^{2}$ Department of Rheumatology, Royal North Shore Hospital and Northern Clinical School, University of Sydney, Sydney, Australia

${ }^{3}$ NHMRC Clinical Trials Centre, University of Sydney, Sydney, Australia

Correspondence to Dr Sarah Rubia Robbins; sarah.meneses@sydney.edu.au

\section{ABSTRACT}

Introduction Current guidelines recommend tailored interventions to optimise knee osteoarthritis $(0 \mathrm{~A})$ management. However, models of care still have a 'one size fits all' approach, which is suboptimal as it ignores patient heterogeneity. This study aims to compare a stepped care strategy with standard care for overweight and obese persons with medial tibiofemoral $O A$.

Methods and analysis Participants will be randomised into two groups (85 each). The intervention will receive a diet and exercise programme for 18 weeks in the first step of the study. Disease remission will then be assessed using the Patient Acceptable Symptom State (PASS). PASS is defined as the highest level of symptom beyond which patients consider themselves well and takes into account pain intensity, patient's global assessment of disease activity and degree of functional impairment. In the second step, participants in remission will continue with diet and exercise. If remission is not achieved, participants will be assigned in a hierarchical order to cognitive behavioural therapy, knee brace or muscle strengthening for 12 weeks. The intervention will be decided based on their clinical presentation for symptoms of depression and varus malalignment. Participants without depression or varus malalignment will undertake a muscle strengthening programme. The control group will receive educational material related to $0 \mathrm{~A}$ management. Main inclusion criteria are age $\geq 50$ years, radiographic medial tibiofemoral $0 \mathrm{~A}$, body mass index (BMI) $\geq 28 \mathrm{~kg} / \mathrm{m}^{2}$, knee pain $\geq 40$ (Visual Analogue Scale, $0-100)$, PASS $(0-100)>32$ for pain and global assessment, and 31 for functional impairment. Outcomes will be measured at 20 -week and 32 -week visits. The primary outcome is disease remission at 32 weeks. Other outcomes include functional mobility; patient-reported outcomes; BMl; waist-hip ratio; quadriceps strength; symptoms of depression, anxiety and stress; and knee range of motion. The analysis will be performed according to the intention-to-treat principle.

Ethics and dissemination The local ethics committee approved this protocol (HREC/14/HAWKE/381). Dissemination will occur through presentations at international conferences and publication in peer-reviewed journals.

Trial registration number ACTRN12615000227594.
Strengths and limitations of this study

To our knowledge, this is the first randomised clinical trial in knee osteoarthritis investigating the efficacy of a stepped care approach.

- Most studies focus on achieving a minimal clinically important improvement. Instead, we will focus on whether the proposed treatment is sufficient to allow participants to reach disease remission.

- Interventions included in this study are usually easily available in practice which facilitates wider implementation.

- We will use results from the subgroup analysis for hypothesis generation only, as we did not power the study for these analyses.

We will not measure adherence to treatments.

\section{INTRODUCTION}

Knee osteoarthritis (KOA) is an ongoing public health challenge with age and obesity as major risk factors. ${ }^{1}$ In 2010 , the prevalence of symptomatic KOA was estimated to be $3.8 \%$ worldwide. $^{2}$ The Global Burden of Disease 2010 Study $^{2}$ ranked it as the 11th top contributor to global disability. Additionally, KOA ranked as the 38th disease with highest overall burden out of 291 health conditions studied. These metrics are projected to worsen with ageing populations and increasing rates of obesity. ${ }^{3}$

KOA is a heterogeneous disorder. ${ }^{4}$ It can affect one or more knee compartments (patellofemoral, lateral and medial tibiofemoral). Also, several factors may influence the perception of pain and play a role in the progression of the disease. ${ }^{5-7}$ This complex condition requires that health practitioners take into account the individual presentation when planning treatment.

Clinical practice guidelines (CPGs) for KOA emphasise the need to tailor interventions to individuals to optimise patient 
outcomes. ${ }^{8-12}$ Despite such recommendations, there is no accepted method for classifying people with KOA into clinically meaningful subgroups that can be used to target treatment. Consequently, the current clinical model uses a 'one size fits all' approach, which is suboptimal as it ignores interpatient heterogeneity. In this context, stepped care strategies have been advocated to enhance the care of heterogeneous chronic conditions such as KOA. ${ }^{13}$

The stepped-care approach is commonly employed in other chronic conditions. ${ }^{14}{ }^{15}$ It has been used in observational studies with the aim of developing and implementing an evidence-based model of care for KOA..$^{1316-19}$ The strategy implies that higher steps, comprised of more complex interventions, should only be considered if previous steps failed to produce satisfactory results. This intervention model has been shown to provide adequate, cost-effective care. ${ }^{20}$ To date, there are no randomised controlled trials (RCTs) in KOA testing stepped interventions. With this in mind, we developed an RCT of a stepped care approach for overweight and obese people with medial tibiofemoral osteoarthritis $(\mathrm{OA})$.

CPGs strongly recommend diet and exercise to all overweight and obese patients with KOA. ${ }^{8-12}$ Thus, the first step will be composed of these interventions. The second step will take into account the presence of depression, knee joint malalignment and muscle weakness. Interventions targeting these factors will be offered to participants who did not reach an acceptable outcome after the first step. Depression, joint malalignment and muscle weakness are well known modifiable factors of poor prognosis and have been shown to have undesirable effects on clinical outcomes of patients with KOA. ${ }^{21}$

Besides its methodology, another novelty of our study is the use of the Patient Acceptable Symptom State (PASS) to assess clinical disease remission. The PASS is defined as the highest level of symptom beyond which patients consider themselves well. ${ }^{22}$ Rather than focusing on the state 'feeling better' we will focus on whether the proposed treatment is sufficient to allow participants to be 'feeling well'. Therefore, the aim of this study is to compare a stepped care treatment programme with usual care for a group of overweight and obese people with medial tibiofemoral OA. Ultimately, this trial should permit the development of a clinical algorithm to tailor individual treatment.

\section{METHODS AND ANALYSIS}

\section{Trial design}

STrEAMline is designed as an RCT, assessor blinded, parallel, two-arm superiority trial with a 1:1 allocation ratio. The trial will be conducted at a single centre in Australia. The interventions will take place from baseline to 32 weeks, and assessments will occur at baseline, 20 weeks and 32 weeks (figure 1). The protocol was designed

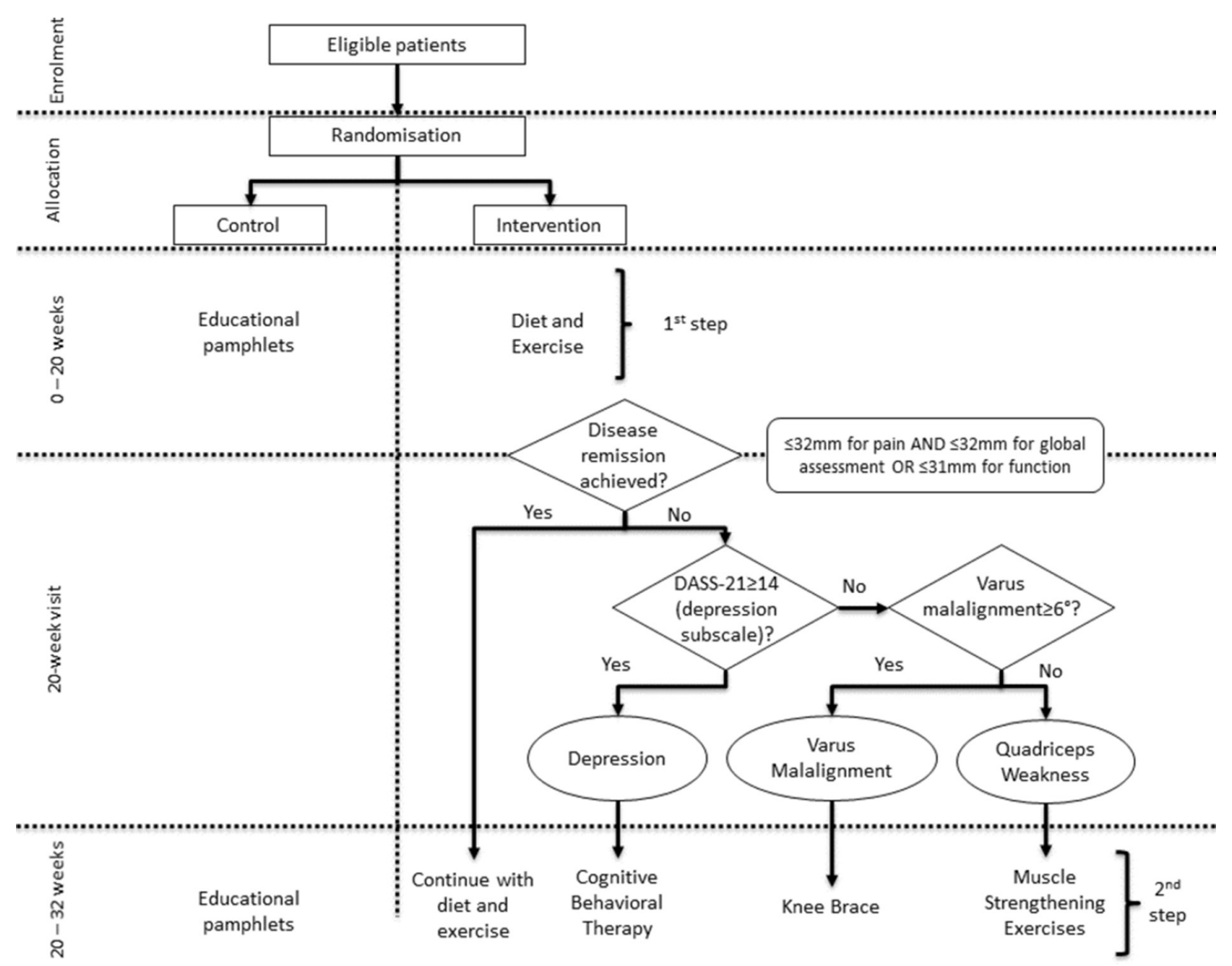

Figure 1 Study flow. DASS-21: Depression, Anxiety and Stress Scale. 
by the principles of the Declaration of Helsinki and according to the standard protocol items: recommendations for interventional trials (SPIRIT) recommendations for reporting protocols. ${ }^{23}$

\section{Participants}

Participants will be recruited from the community through advertisements on noticeboards, waiting-room walls, newsletters and social media, such as Facebook and Twitter.

\section{Inclusion criteria}

Participants will be eligible for the study if they meet all the following inclusion criteria: age $\geq 50$ years; body mass index $(\mathrm{BMI}) \geq 28 \mathrm{~kg} / \mathrm{m}^{2}$ (requirement for the diet and exercise programme); knee pain or stiffness for most of the past 30 days; average pain on the Visual Analogue Scale (VAS) $\geq 40$ out of $100 \mathrm{~mm}$ over the last 48 hours prior to the screening visit. PASS scores must be greater than 32 for pain, 32 for patient's global assessment of disease activity and 31 for function impairment out of $100 \mathrm{~mm}$ (please refer to the Primary outcome section for further information on PASS).

A trained rheumatologist will assess radiographic evidence of disease in the medial tibiofemoral compartment. The presence of a definite osteophyte will be used to determine radiographic evidence of KOA (KellgrenLawrence grade, KLG) $\geq 2 .{ }^{24}$ The presence of joint space narrowing (JSN) in the medial tibiofemoral compartment will be determined with reference to the Osteoarthritis Research Society International atlas. ${ }^{25}$ To assure medial compartment involvement, the medial JSN will have to be greater than the lateral JSN. If the pain scores are the same for both knees, participants will be asked to nominate the worse knee (ie, the one that causes more problems to the participant), which will be included as the index joint.

\section{Exclusion criteria}

Participants will be excluded if they fulfil any of the following criteria: radiographic evidence of predominant patellofemoral disease; radiographic evidence of predominant lateral tibiofemoral disease; any other knee pathology that is likely to be contributing to their knee pain (eg, pes anserine bursitis, referred knee pain from conditions of the back or hip, presence of inflammatory rheumatic diseases such as rheumatoid arthritis or gout); people currently on the waiting list for knee joint replacement or already being treated with any of the study interventions.

\section{Study procedures}

\section{Preliminary screening}

The key inclusion/exclusion criteria will be assessed through a preliminary screening that will occur by phone or online. People who pass this preliminary screening will be invited to attend a screening visit to determine eligibility.

\section{Screening visit}

Prior to the visit, participants will receive verbal and written information about the trial. At the beginning of the visit, a written informed consent will be obtained. We will collect demographic and clinical data regarding the key inclusion/exclusion criteria to confirm the participant's eligibility. The presence of comorbid diseases will be assessed using the Self-Administered Comorbidity Questionnaire. ${ }^{26}$ Table 1 contains the schedule of enrolment and assessments.

\section{Baseline visit}

The baseline visit will happen on the same day as screening, after confirming the radiographic evidence of disease in the medial tibiofemoral compartment. The primary and secondary outcomes will be collected at this point.

\section{Follow-up visits}

Follow-up visits will occur at 20 weeks and 32 weeks.

\section{Randomisation and allocation concealment}

Individuals who consent to take part in the study and fulfil all study eligibility criteria will be assigned to either the intervention group or the control group at the end of the baseline visit. Randomisation will be stratified according to KLG (KLG 2, 3 and 4). Participants will be allocated to treatment or control according to a computer generated randomisation list (Stata V.10.1). We will use a 1:1 ratio and random blocks of size 2, 4 and 6 .

The allocation sequence will be concealed from the researchers enrolling and assessing participants in sequentially numbered opaque and sealed envelopes. Envelopes will be stored in a locked drawer and will be opened by the study coordinator. A statistician will prepare the sequence generation and an external investigator not involved in the trial will arrange the envelopes.

\section{Blinding}

An assessor blinded to the treatment allocation will conduct all clinical assessments. Participants will be instructed not to disclose any information about the treatment and not to bring any study items during the follow-up assessments to reduce the potential for unblinding. The physiotherapist executing and supervising the treatments will not be blinded to the group allocation. Due to the two-step intervention process only occurring in one randomised arm, it will not be possible to blind the statistician conducting the analysis. Group assignment will be immediately unblinded if deemed necessary by the chief investigator in the case of serious adverse events potentially related to the study.

\section{Outcomes}

The outcomes used in this study will be measured using validated instruments and assessed at baseline, and 20-week and 32-week visits (table 1). 
Table 1 Schedule of enrolment, interventions and assessments

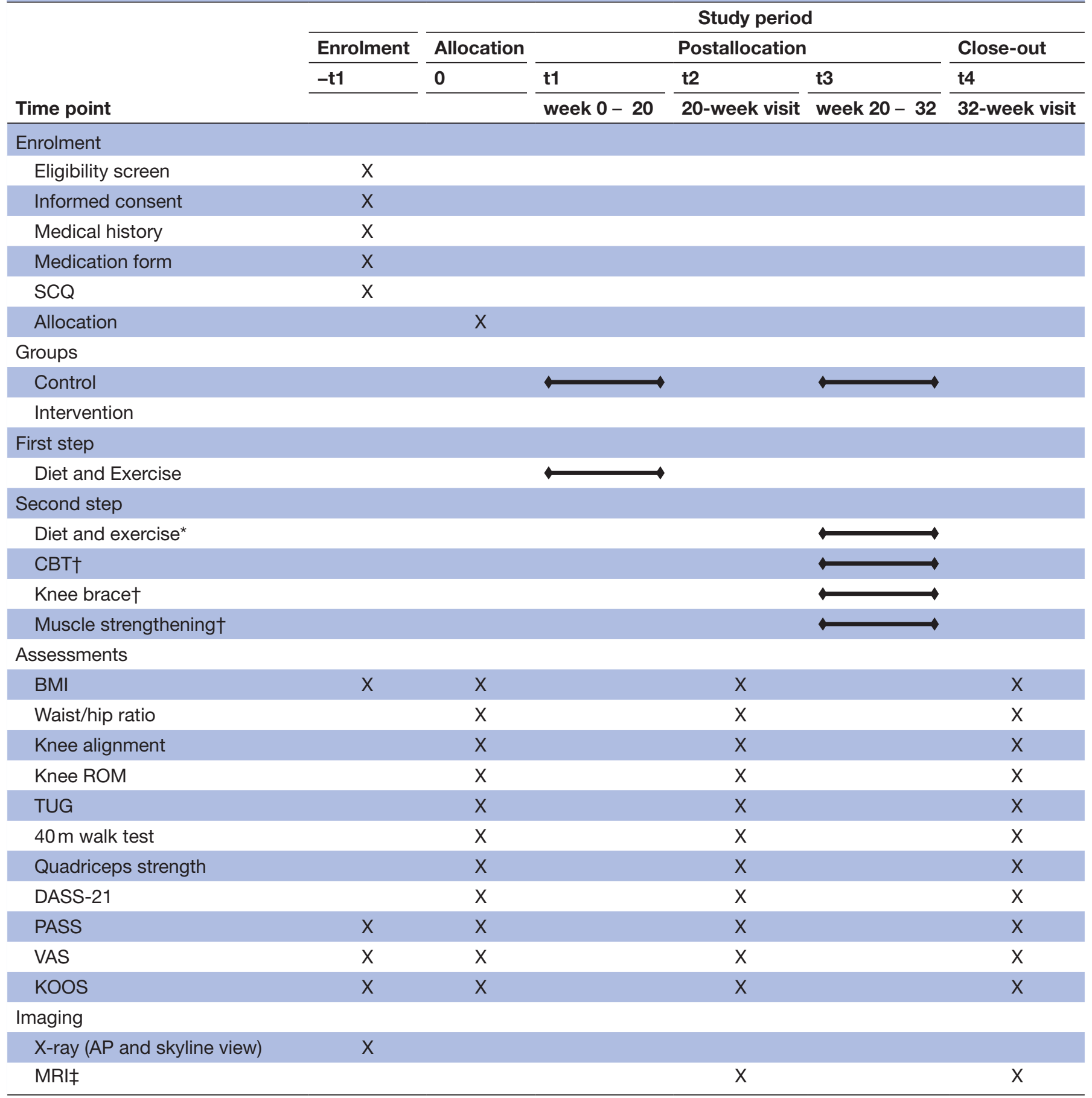

*If participant achieved disease remission.

†lf participant did not achieve disease remission.

fFor participants in the knee brace subgroup.

AP, anteroposterior; BMI, body mass index; CBT, cognitive behavioural therapy; DASS-21, Depression, Anxiety and Stress Scale; KOOS, Knee Injury and Osteoarthritis Outcome Score; PASS, Patient Acceptable Symptom State; ROM, range of motion; SCQ, Self-Administered Comorbidity Questionnaire; TUG, Timed Up and Go; VAS, Visual Analogue Scale.

The last part of the visit will focus on radiographic assessment of knee osteoarthritis. Referral for a knee X-ray will be provided for the participants who do not have a recent one (less than 12 months). Participants will only be provided with a referral for a knee X-ray if they meet all other inclusion criteria. Standing AP and skyline views will be acquired by Castlereagh Imaging on the same day and the cost will be covered by the study.

\section{Primary outcome}

The primary outcome will be disease remission (yes/ no) at 32 weeks measured using PASS. ${ }^{22}$ PASS comprises three domains: pain intensity, patient's global assessment of disease activity and function impairment. To achieve disease remission at 32 weeks, patients must 
achieve pain remission as well as remission for patients' global assessment of disease activity and/or physical function.

Pain intensity will be measured on a $0-100 \mathrm{~mm}$ VAS, in which 0 represents no pain and 100 the worst possible pain, considering the last 48 hours and remission will be defined as a score $\leq 32 \mathrm{~mm}$. Patient's global assessment of disease activity will be assessed with the question 'Considering all the ways your arthritis affects you, how have you been during the last 48 hours?'. It will be measured on a $0-100 \mathrm{~mm}$ VAS ( 0 is very well, 100 is very poor) and remission will be defined as a score $\leq 32 \mathrm{~mm}$. Function impairment will be derived from the physical function subscale of the Knee injury and Osteoarthritis Outcome Score $(\mathrm{KOOS})^{27}$ and converted to a $0-100$ score. Remission will be defined as a score $\leq 31 \mathrm{~mm}$.

\section{Secondary outcomes}

The following secondary outcome measures will be assessed at 32 weeks and calculated as the change from baseline: knee pain measured by VAS; ${ }^{28}$ patient-reported outcomes assessed by KOOS; ${ }^{27}$ physical performance measured by the Timed Up and Go test ${ }^{29}$ and fast-paced walk test $40 \mathrm{~m} ;{ }^{30}$ BMI $\left(\mathrm{BMI}=\right.$ weight $(\mathrm{kg}) /$ height $^{2}(\mathrm{~m})$ ); waist-hip ratio calculated using waist and hip circumference; quadriceps strength (in $\mathrm{Nm} / \mathrm{kg}$ ) measured by isometric knee extensor strength test using a force gauge; severity of depression, anxiety and stress symptoms measured using the Depression, Anxiety and Stress Scale (DASS-21) $;{ }^{31}$ knee range of motion measured by goniometry of knee extension and flexion. Frontal plane knee alignment will also be measured at all three time points by goniometry.

Treatment response will be measured by perceived ratings of change (much worse; moderately worse; slightly worse; no change; slightly better; moderately better and much better $)^{32}$ and by the Outcome Measures in Rheumatology-Osteoarthritis Research Society International (OMERACT-OARSI) criteria for a meaningful change. ${ }^{33}$

\section{Interventions}

Control group

The control group will receive educational pamphlets about OA and its management at each assessment (baseline, 20-week and 32-week). These fact sheets will be based on the MyJointPain website, ${ }^{34}$ which was developed by Arthritis Australia and medical experts, ${ }^{35}$ and is a source of reliable information about disease management for individuals with OA. It provides users with a collection of OA healthcare services to allow patients to determine who is best suited to manage their illness. Participants will be encouraged to access the website for extra educational material.

\section{Intervention group - first step}

The intervention group will be based on a stepped care approach. The first step will be a diet and exercise regime based on the Healthy Weight For Life (HWFL) programme,${ }^{36}$ which consists of an 18-week, multistaged programme.

There are three stages of 6 weeks: motivational weight loss, consolidation phase and short-term maintenance phase.

Phase 1 (motivational weight loss): participants will receive and consume a nutritionally complete very low-calorie diet meal replacement (KicStart, food for the particular medical purpose) for two meals per day, in combination with controlled portions and 'free foods' (eg, berries, leafy greens). This initial phase was designed to result in a significant early weight loss. The rapid weight loss is intended to improve motivation and adherence.

Phase 2 (consolidation weight loss): one meal replacement per day, with 'free foods' and portion-controlled lunch and dinner, aimed to wean off meal replacements progressively.

Phase 3 (short-term weight maintenance): portion-controlled whole foods for breakfast, lunch and dinner, and 'free foods'.

Throughout the programme, participants will be given a choice of meal replacement flavours and provided access to recipes for low fat and low glycaemic index meals. A portion-control eating plan and healthy lifestyle education will be provided, as well as coaching and personalised telephone motivation, support and advice.

The HWFL exercise programme focuses on balance, strengthening and flexibility of quadriceps, calf, hamstrings, gluteal, hip flexor and abductor muscles. It is a home-based intervention for which the participants will receive resources developed in collaboration with physiotherapists. The resources and tools included are a manual with step-by-step instructions for 42 different exercises across three levels, a digital versatile disc (DVD) demonstrating and explaining each of the 42 exercises, resistance exercise tube and a hot pack and a cold pack for pain management. Exercises should be performed at least three times per week, ideally separating each session by a day or two. Participants will be asked to only exercise within their physical capabilities and to not continue exercising with increasing pain or pain that is moderate to severe.

Phase 1: participants will be encouraged to maintain a routine of three sets of $10 \mathrm{~min}$ of physical daily activity (such as walking or water based), and between 12 and 15 level 1 exercises.

Phase 2: will require $30 \mathrm{~min}$ (or more) of daily physical activity (either land or water based), and between 12 and 15 level 2 exercises.

Phase 3: will require $30 \mathrm{~min}$ (or more) of physical activity daily (either land based or water based) or the use of a pedometer to target 10000 steps per day and between 12 and 15 level 3 exercises.

Intervention group - second step

Disease remission will be assessed at 20 weeks using PASS. Participants in remission will continue with diet and exercise until reassessment at 32 weeks. Participants who do 
not achieve remission will step up into one of the three treatment subgroups for 12 weeks based on their clinical presentation for symptoms of depression and varus malalignment. Participants who do not fit the criteria for depression or varus malalignment will undertake a muscle strengthening programme.

\section{Depression}

We will use DASS-21 to assess symptoms of depression. DASS-21 comprises three subscales namely depression, anxiety and stress. ${ }^{31}$ Participants with a DASS-21 Score $\geq 28$ for depression, which is defined as severely depressed, will be referred for immediate psychiatric evaluation. Participants who score $\geq 14$ on the depression subscale, defined as presenting moderate symptoms of depression, will enter the depression treatment arm.

These participants will be referred to the Clinical Research Unit for Anxiety and Depression (CRUfAD). ${ }^{37}$ CRUfAD offers an online cognitive behavioural therapy course for the treatment of depression. This course involves six online lessons to be completed over a 12-week period. The training sessions provide a structured learning plan, downloadable materials and guidance from a dedicated clinical psychologist expert in the treatment of depression.

\section{Varus malalignment}

The gold standard measure of knee alignment angle uses a full-limb radiograph to measure the mechanical axis. However, it has been shown to be possible to estimate the mechanical axis through the anatomical axis which only requires a traditional anteroposterior knee radiograph. ${ }^{38}$ According to this method, the mechanical axis is obtained by subtracting 4.21 from the anatomical axis. The reliability of the knee alignment angle measurements will be assessed and reported.

Participants who have varus malalignment $\geq 6$ degrees will receive the Unloader FIT knee brace, ${ }^{39}$ which will be fitted by a certified trained practitioner respecting the participant's knee circumference measurements $(15 \mathrm{~cm}$ above and $15 \mathrm{~cm}$ below the mid-patella). The participants will also receive proper instructions regarding the appropriate use and maintenance of the device.

For the first 2 days, participants will be advised to wear the brace for a minimum of 2 hours to allow time for adaptation. After that, they will be instructed to use the brace daily during physical activities for a minimum of 6 hours. Össur will supply the knee brace free of charge. Participants will be given the option to keep the brace at the conclusion of the study.

Non-contrast-enhanced MRIs will be obtained for this subgroup of participants before and after the step 2 phase. The aim is to determine the influence of the brace in decreasing the load on the medial compartment of the knee and its effect on bone marrow lesions, assessed on MRI using the MRI Osteoarthritis Knee Score. ${ }^{40}$ The MRIs will be obtained at Castlereagh Imaging and will be free of charge to participants. Ideally, the first MRI will

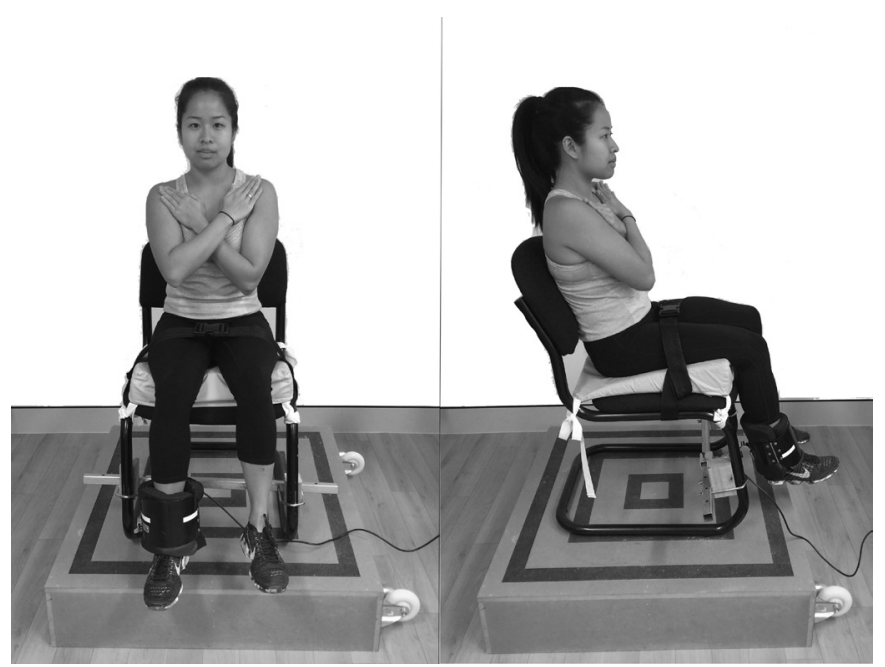

Figure 2 Chair strength test.

be scheduled as close as possible to the 20-week visit, and the brace will be fitted on the same day as the MRI. The second MRI will be scheduled on the same day as the 32-week visit.

\section{Quadriceps weakness}

Quadriceps strength will be measured by an isometric knee extensor strength test using a digital force gauge. Participants will be seated in a standard chair with the knee joint fixed at $90^{\circ}$ of flexion. The force gauge will be attached to the chair by a gripping jaw. The system is illustrated in figure 2. Due to the lack of normative data for quadriceps strength in people with KOA, participants who do not fit the criteria for depression or varus malalignment will undertake a muscle strengthening programme.

The programme will follow the quadriceps strengthening programme outlined by Bennell et a $t^{11}$ comprised of five distinct non-weight-bearing exercises. The participants will attend seven visits with a trained physiotherapist over 12 weeks for exercise prescription, monitoring and progression. Each supervised exercise session will last approximately $30 \mathrm{~min}$. Participants will be instructed to perform the prescribed exercises at home five times per week.

\section{Participants with multiple problems identified at 20 weeks}

The following decision-making matrix will be implemented individually for those participants who fit the criteria for entry into more than one treatment arm, at the 20-week assessment:

Depression >varus malalignment $>$ quadriceps weakness.

The decision-making matrix was developed in this way due to the level of impact each of these problems poses to achieve remission of OA. A recent study found that depression adversely affects the quality of life of patients with KOA ${ }^{42}$ For this reason, it is imperative that all participants with symptoms of depression receive adequate treatment.

Ranking varus malalignment ahead of quadriceps weakness was decided with the intent that correcting existing malalignment will help to improve the mechanical 
functioning of the joint, and in doing so, it will allow the muscles that act on the knee joint to function correctly.

\section{Participant safety and withdrawal}

Risk management and safety

Any participant who is identified as suffering from severe depression will be withdrawn from the study and referred to a mental health professional.

Muscle strength testing and strengthening exercises may be performed throughout this study, which carries a slight risk of injury. In the event of harm, participants will be withdrawn from the study and will be referred to appropriate health professionals (eg, physiotherapists, physicians). All serious adverse events will be reported.

\section{Handling of withdrawals}

Should a participant withdraw from the study, they will have their reasons for withdrawal recorded, and their scheduled assessments will be maintained regardless of withdrawal status by our data security and handling protocol. Participants who withdraw from the study will not be replaced.

\section{Statistical methods}

\section{Sample size estimation and justification}

A $25 \%$ difference in disease remission rate between the two groups would make the programme worthwhile. For this trial, we estimate that $45 \%$ of participants in the control group and $70 \%$ of participants in the intervention group will have disease remission at 32 weeks. With 68 subjects in each group, there will be sufficient power $(80 \%)$ to detect a statistically significant difference $(\mathrm{P}<0.05)$. After incorporating a dropout rate of $20 \%$, a total of 170 will be needed for the study.

\section{Statistical analysis}

Data will be analysed according to the intention-to-treat principle. Baseline demographic characteristics and patient-reported outcome scores will be summarised using mean and SD for continuous variables or median (quartiles) if the distribution is skewed. Counts with percentages will be presented for categorical variables.

The primary outcome of interest is the proportion of patients who achieve remission at 32 weeks. The percentage of patients who achieve remission at 32 weeks will be presented as frequency (percentage) by treatment arm, the difference in proportions will be summarised as mean (95\% CI) and analysed using a two-proportion Z-test. Logistic regression will be used to adjust treatment effect for the pertinent baseline demographic and clinical outcomes.

For continuous outcomes, the mean scores (SD) will be presented at each time point by treatment group. The between-group difference in mean change from baseline with 95\% CI will be presented for all primary and secondary outcomes and compared using an independent t-test or the Wilcoxon rank-sum test as appropriate. Categorical outcomes will be examined by $\chi^{2}$ test or Fisher's exact test if expected cell counts are small.
Logistic regression will be used to assess differences between treatments in perceived response and OMERACT-OARSI responder's criteria. Both unadjusted models and models adjusting for age, gender, BMI and KLG will be performed.

A generalised estimating equation model will be fitted to investigate the effect of interventions over time, including a treatment-by-time interaction term alongside treatment and time main effects. As an exploratory analysis and if numbers allow, pain and function scores will be plotted over time for all possible study arms (ie, OA education and the diet and exercise regime subgroups) and analysed using ANOVA.

Post hoc subgroup analyses will be performed examining whether there is heterogeneity in treatment effect according to gender, age group, KLG, BMI, the presence of bilateral $\mathrm{OA}$ and the number of comorbidities. Logistic regression models will be fitted including a treatment-by-subgroup interaction term alongside treatment and subgroup as main effects.

The association of weight loss and pain will be assessed using regression analysis and adjusting for the treatment group. For the varus malalignment subgroup, we will investigate the influence of the knee brace use on bone marrow lesions assessed by a pre-MRI and a post-MRI.

\section{Data security and handling}

Study data will be collected and managed using the Research Electronic Data Capture which is hosted at University of Sydney. ${ }^{43}$ Backups will be kept in password-protected electronic files. The privacy, security and ownership of the research data will be maintained and will not be stored or accessible by another organisation. During and after completion of the study, any paper forms will be retained at the study coordinator's office in a locked filing cabinet, and offices are restricted to card access only.

In compliance with the New South Wales State Records Act, the archiving period for clinical research records will be 15 years. After this time, the electronic files will be deleted. The information technology department will be involved to ensure the deletion and records are not retrievable from any source. The paper forms will be destroyed after this period.

\section{Ethics and dissemination}

Any protocol modification will be sent to review by the research ethics committee and will be amended at the trial registry. We also obtained consent to publish the photographs in figure 2 from the person pictured. Dissemination is planned to occur through presentations at international conferences, publication in peerreviewed journals and social media. No information which could lead to the identification of a participant will be included in the dissemination of results. Only fully nonidentifiable data will be presented when disseminating results. All collected data will also be stored in a locked cabinet throughout the course of the study. 
Acknowledgements The authors thank Prima Health and Össur for providing the HWFL programme and the knee braces, respectively, free of charge. The authors also thank Professor Kim Bennell for the chair system for strength assessment. The authors also thank the consumer focus group for their contribution to the study design and feedback.

Contributors SRR, LRSM, VLJ and DJH contributed to study conception and design. SRR, HU and LAD contributed to data collection. DJH attained project funding. SRR and LRSM drafted the first version of the manuscript. LRSM is responsible for data management. RA performed the statistical analysis. All authors revised the protocol critically for important intellectual content and read and approved the final version of the protocol.

Funding This work is supported by the National Health and Medical Research Council (NHMRC) programme grant (grant number APP1091302) and sponsored by the University of Sydney.

Competing interests DJH reports grants from National Health and Medical Research Council, non-financial support from Prima Health, non-financial support from Össur, personal fees from Merck Serono, personal fees from National Health and Medical Research Council, during the conduct of the study; personal fees from Flexion, personal fees from Tissuegene, personal fees from Nestle, personal fees from Merck Serono, outside the submitted work. The remaining authors report grant from the National Health and Medical Research Council, non-financial support from Prima Health and non-financial support from Össur, during the conduct of the study.

Patient consent Obtained.

Ethics approval Human Research Ethics Committee (HREC) of the Northern Sydney Local Health District (HREC/14/HAWKE/381)

Provenance and peer review Not commissioned; externally peer reviewed.

Open Access This is an Open Access article distributed in accordance with the Creative Commons Attribution Non Commercial (CC BY-NC 4.0) license, which permits others to distribute, remix, adapt, build upon this work non-commercially, and license their derivative works on different terms, provided the original work is properly cited and the use is non-commercial. See: http://creativecommons.org/ licenses/by-nc/4.0/

C Article author(s) (or their employer(s) unless otherwise stated in the text of the article) 2017. All rights reserved. No commercial use is permitted unless otherwise expressly granted.

\section{REFERENCES}

1. Ackerman IN, Osborne RH. Obesity and increased burden of hip and knee joint disease in Australia: results from a national survey. BMC Musculoskelet Disord 2012;13:254.

2. Cross M, Smith E, Hoy D, et al. The global burden of hip and knee osteoarthritis: estimates from the global burden of disease 2010 study. Ann Rheum Dis 2014;73:1323-30.

3. Hunter DJ, Schofield D, Callander E. The individual and socioeconomic impact of osteoarthritis. Nat Rev Rheumatol 2014;10:437-41.

4. Bruyère $\mathrm{O}$, Cooper $\mathrm{C}$, Arden $\mathrm{N}$, et al. Can we identify patients with high risk of osteoarthritis progression who will respond to treatment? A focus on epidemiology and phenotype of osteoarthritis. Drugs Aging 2015;32:179-87.

5. Bastick AN, Belo JN, Runhaar J, et al. What are the prognostic factors for radiographic progression of knee osteoarthritis? A metaanalysis. Clin Orthop Relat Res 2015;473:2969-89.

6. Neogi T. The epidemiology and impact of pain in osteoarthritis. Osteoarthritis Cartilage 2013;21:1145-53.

7. Bastick AN, Runhaar J, Belo JN, et al. Prognostic factors for progression of clinical osteoarthritis of the knee: a systematic review of observational studies. Arthritis Res Ther 2015;17:152

8. National Institute for Health and Clinical Excellence. Guidance. In: Osteoarthritis: care and management in adults. London: National Institute for Health and Clinical Excellence, 2014.

9. Fernandes L, Hagen KB, Bijlsma JW, et al. EULAR recommendations for the non-pharmacological core management of hip and knee osteoarthritis. Ann Rheum Dis 2013;72:1125-35.

10. Hochberg MC, Altman RD, April KT, et al. American college of rheumatology 2012 recommendations for the use of nonpharmacologic and pharmacologic therapies in osteoarthritis of the hand, hip, and knee. Arthritis Care Res 2012;64:465-74.

11. Jevsevar DS. Treatment of osteoarthritis of the knee: evidence-based guideline, 2nd edition. J Am Acad Orthop Surg 2013;21:571-6.
12. McAlindon TE, Bannuru RR, Sullivan MC, et al. OARSI guidelines for the non-surgical management of knee osteoarthritis. Osteoarthritis Cartilage 2014;22:363-88.

13. Porcheret M, Jordan K, Croft P. Treatment of knee pain in older adults in primary care: development of an evidence-based model of care. Rheumatology 2007;46:638-48.

14. Olivier HE, Jamero D. Implementation of a hypertension clinic using a streamlined treatment algorithm. Am J Health Syst Pharm 2012;69:664-7.

15. Stoop CH, Nefs G, Pommer AM, et al. Effectiveness of a stepped care intervention for anxiety and depression in people with diabetes, asthma or COPD in primary care: a randomized controlled trial. $J$ Affect Disord 2015;184:269-76.

16. Barten DJ, Smink A, Swinkels IC, et al. Factors associated with referral to secondary care in patients with osteoarthritis of the hip or knee after implementation of a stepped-care strategy. Arthritis Care Res 2017;69:216-25.

17. Smink AJ, Bierma-Zeinstra SM, Schers HJ, et al. Non-surgical care in patients with hip or knee osteoarthritis is modestly consistent with a stepped care strategy after its implementation. Int J Qual Health Care 2014;26:490-8.

18. Smink AJ, Dekker J, Vliet Vlieland TPM, et al. Health care use of patients with osteoarthritis of the hip or knee after implementation of a stepped-care strategy: an observational study. Arthritis Care Res 2014;66:817-27.

19. Smink AJ, van den Ende $\mathrm{CH}$, Vliet Vlieland TP, et al. Effect of stepped care on health outcomes in patients with osteoarthritis: an observational study in Dutch general practice. Br J Gen Pract 2014;64:e538-44.

20. Ho FY, Yeung WF, Ng TH, et al. The efficacy and cost-effectiveness of stepped care prevention and treatment for depressive and/or anxiety disorders: a systematic review and meta-analysis. Sci Rep 2016;6:29281.

21. Kittelson AJ, Stevens-Lapsley JE, Schmiege SJ. Determination of pain phenotypes in knee osteoarthritis: a latent class analysis using data from the osteoarthritis initiative. Arthritis Care Res 2016;68:612-20.

22. Tubach F, Ravaud P, Baron G, et al. Evaluation of clinically relevant states in patient reported outcomes in knee and hip osteoarthritis: the patient acceptable symptom state. Ann Rheum Dis 2005:64:34-7.

23. Chan AW, Tetzlaff JM, Altman DG, et al. SPIRIT 2013 statement: defining standard protocol items for clinical trials. Rev Panam Salud Publica 2015;38:506-14.

24. Kellgren JH, Lawrence JS. Radiological assessment of osteoarthrosis. Ann Rheum Dis 1957;16:494-502.

25. Altman RD, Gold GE. Atlas of individual radiographic features in osteoarthritis, revised. Osteoarthritis Cartilage 2007;15(Suppl A):A1-56.

26. Sangha $\mathrm{O}$, Stucki $\mathrm{G}$, Liang $\mathrm{MH}$, et al. The self-administered comorbidity questionnaire: a new method to assess comorbidity for clinical and health services research. Arthritis Rheum 2003;49:156-63.

27. Collins NJ, Prinsen CA, Christensen R, et al. Knee injury and osteoarthritis outcome score (KOOS): systematic review and meta-analysis of measurement properties. Osteoarthritis Cartilage 2016;24:1317-29.

28. Revill SI, Robinson JO, Rosen M, et al. The reliability of a linear analogue for evaluating pain. Anaesthesia 1976;31:1191-8.

29. Piva SR, Fitzgerald GK, Irrgang JJ, et al. Get up and go test in patients with knee osteoarthritis. Arch Phys Med Rehabil 2004:85:284-9.

30. Wright AA, Cook CE, Baxter GD, et al. A comparison of 3 methodological approaches to defining major clinically important improvement of 4 performance measures in patients with hip osteoarthritis. J Orthop Sports Phys Ther 2011:41:319-27.

31. $\mathrm{Ng} \mathrm{F}$, Trauer T, Dodd S, et al. The validity of the 21-item version of the depression anxiety stress scales as a routine clinical outcome measure. Acta Neuropsychiatr 2007;19:304-10.

32. Revicki D, Hays RD, Cella D, et al. Recommended methods for determining responsiveness and minimally important differences for patient-reported outcomes. J Clin Epidemiol 2008;61:102-9.

33. Pham T, van der Heijde D, Altman RD, et al. OMERACT-OARSI initiative: osteoarthritis research society international set of responder criteria for osteoarthritis clinical trials revisited. Osteoarthritis Cartilage 2004;12:389-99.

34. MyJointPain. https://www.myjointpain.org.au/

35. Arthritis Australia. http://www.arthritisaustralia.com.au/

36. Health Weight For Life. https://healthyweightforlife.com.au/ 
37. Clinical Research Unit for Anxiety and Depression (CRUfAD). https:// crufad.org/

38. Kraus VB, Vail TP, Worrell T, et al. A comparative assessment of alignment angle of the knee by radiographic and physical examination methods. Arthritis Rheum 2005;52:1730-5.

39. Unloader® FIT. http://www.ossur.com.au/oa-solutions/oa-products/ oa-knee/oa-knee-braces/unloader-fit

40. Hunter DJ, Guermazi A, Lo GH, et al. Evolution of semi-quantitative whole joint assessment of knee OA: MOAKS (MRI Osteoarthritis Knee Score). Osteoarthritis Cartilage 2011;19:990-1002.
41. Bennell KL, Egerton T, Wrigley TV, et al. Comparison of neuromuscular and quadriceps strengthening exercise in the treatment of varus malaligned knees with medial knee osteoarthritis: a randomised controlled trial protocol. BMC Musculoskelet Disord 2011;12:276.

42. Sharma A, Kudesia P, Shi Q, et al. Anxiety and depression in patients with osteoarthritis: impact and management challenges. Open Access Rheumatol 2016;8:103-13.

43. Research Electronic Data Capture (REDCap). https://redcap.sydney. edu.au/ 